\title{
Role of IL-6/JAK/STAT pathway in inducing vascular insulin resistance
}

\author{
Aswath Balakrishnan*, Kapaettu Satyamoorthy, Manjunath B Joshi \\ From International Conference on Human Genetics and 39th Annual Meeting of the Indian Society of \\ Human Genetics (ISHG) \\ Ahmadabad, India. 23-25 January 2013
}

\section{Introduction}

Insulin resistance is a hall mark of metabolic disorders. Studies have demonstrated that inflammatory regulator interleukin-6 (IL-6) plays an important role in disruption of IR/Akt/eNOS signaling pathway resulting vascular insulin resistance. Accumulating evidences suggests a significant role of epigenetic mechanisms such as DNA methylation in progression of metabolic disorders. Hence the present study aimed to understand the role of epigenetic mechanisms involved during IL-6 induced vascular insulin resistance and its consequences in cardiovascular diseases.

\section{Materials and methods}

Human umbilical vein endothelial cells (HUVEC) and Human dermal microvascular endothelial cells (HDMEC) were used for this study. Endothelial cells were treated in presence or absence IL-6 (20ng/ml) for 36 hours and followed by insulin $(100 \mathrm{nM})$ stimulation for 15 minutes. Levels of phosphorylated- and total Akt served as readout for insulin resistance. To investigate changes in DNA methylation, cells were treated with or without neutrophil conditioned medium (NCM) as a physiological source of inflammation or IL-6 for 36 hours. Genomic DNA was processed for HPLC analysis for methyl cytosine content and cell lysates were analyzed for DNMT1 (DNA (cytosine-5)-methyltransferase 1) and DNMT3A (DNA (cytosine-5)-methyltransferase 3A) levels using immunoblotting.

\section{Results}

Endothelial cells stimulated with insulin exhibited an increase in phosphorylation of Akt ${ }^{\text {ser } 473}$ in serum free conditions but such insulin response was not observed in cells treated with IL-6, suggesting chronic exposure of endothelial cells to IL-6 leads to insulin resistance. HPLC analysis for global DNA methylation resulted in decreased levels of methyl cytosine in cells treated with pro-inflammatory molecules (both by NCM and IL6) as compared to $3.2 \%$ in untreated control to $2 \%$ in treated. Kinetic studies depicted a transient increase DNA methylation at 24 hours which was followed by steep decrease at 36 hours. Subsequently, analysis in cells treated with IL-6 showed a significant decrease in DNMT1 levels but not in DNMT3A. Other pro-inflammatory marker such as TNF- $\alpha$ did not exhibit such changes. Interestingly we also observed Akt phsophorylation refelcetd DNMT1 changes suggesting plausible role of PI3K/Akt signaling axis in regulation of DNMT1 expression.

\section{Conclusion}

Taken together our study suggests that IL- 6 induces vascular insulin resistance and involvement of epigenetic changes.

Published: 21 January 2014

doi:10.1186/1755-8166-7-S1-P96

Cite this article as: Balakrishnan et al:: Role of IL-6/JAK/STAT pathway in inducing vascular insulin resistance. Molecular Cytogenetics 2014 7(Suppl 1):P96. 